\title{
Neutrino-nucleus DIS data and their consistency with nuclear PDFs
}

\author{
Hannu Paukkunen* \\ University of Jyväskylä, Department of Physics, Finland \\ Helsinki Institute of Physics, Finland \\ E-mail: hannu.paukkunen@ jyu.fi
}

\section{Carlos A. Salgado}

University of Santiago de Compostela, Departamento de Física de Partículas and IGFAE, Spain

E-mail: carlos.salgado@usc.es

In this talk, we discuss the compatibility of different deeply inelastic neutrino-nucleus data sets and the universal nuclear PDFs. This is an issue that has lately been investigated by different groups but the conclusions have been surprisingly contradictory. While some studies have found a good overall agreement between the nuclear PDFs and the neutrino data, others have claimed for an incompatibility. Here, we demonstrate that the independent neutrino data sets from $\mathrm{NuTeV}$, CHORUS and CDHSW collaborations differ in the absolute overall normalization and that it is not possible to accurately reproduce all the data simultaneously with a single set of PDFs. Our strategy to overcome this difficulty and allow a consistent use of all neutrino data in global PDF analyses is to normalize the data by the integrated cross-sections thereby cancelling possible inaccuracies in the absolute normalization. Indeed, this brings all data to a surprisingly good mutual agreement underscoring the $\mathrm{x}$-dependence of the nuclear modifications in a model-independent way. The consistency of these data with the present nuclear PDFs is verified by introducing a method to test the effect of a new data set in an existing global fit that performed a Hessian error analysis.

XXI International Workshop on Deep-Inelastic Scattering and Related Subjects 22-26 April, 2013

Marseilles, France

\footnotetext{
*Speaker.
} 


\section{Introduction}

The large- $Q^{2}$ neutrino-nucleus $(v A)$ deep inelastic scattering is an interesting "cross-over" process that can shed light on quite different sides of parton distribution functions (PDFs). In leading order, the partonic content of the nucleon probed in charged-current $v A$ cross-sections can be schematically written as

$$
\frac{d^{2} \sigma^{v A}}{d x d y} \propto(d+s+b)+(1-y)^{2}(\bar{u}+\bar{c}) \quad ; \quad \frac{d^{2} \sigma^{\bar{v} A}}{d x d y} \propto(\bar{d}+\bar{s}+\bar{b})+(1-y)^{2}(u+c),
$$

which should be compared to the corresponding expression for the standard charged-lepton induced neutral-current process

$$
\frac{d^{2} \sigma^{\ell^{ \pm} A}}{d x d y} \propto \frac{4}{9}(u+c+\bar{u}+\bar{c})+\frac{1}{9}(d+s+b+\bar{d}+\bar{s}+\bar{b}) .
$$

Of importance to the free proton analyses is the strange quark PDF that is more pronounced in neutrino reactions than in the charged-lepton process where the already small strange quark PDF is additionally suppressed by the QED coupling. On the other hand, the neutrino data is taken with nuclear targets and should therefore be useful for constraining the nuclear effects in PDFs.

The use of $v A$ data for either purpose relies naturally on the adequacy of the collinear factorization in these processes which many free proton fits take as granted by using these data. However, this assumption has been doubted. In particular, it was reported $[2,3]$ that the $v A$ data from the $\mathrm{NuTeV}$ collaboration [1] prefer quite different nuclear modifications in PDFs as the other existing $\ell^{ \pm} A$ data. Later publication [4] by the same collaboration declared all $v A$ data as incompatible with the $\ell^{ \pm} A$ data. Rather different strategy was adopted in [5], where data from independent neutrino experiments (NuTeV [1], CDHSW [6], CHORUS [7]) were contrasted with the existing nuclear PDFs. While an excellent overall global agreement was found, surprisingly large, beam energy dependent fluctuations in the absolute normalization of the $\mathrm{NuTeV}$ data sample were noticed and suggested to cause the results of [2, 3, 4]. In a recent analysis [8], these $v A$ data were included in a global fit of nuclear PDFs. No difficulties in accommodating these data with other $\ell^{ \pm} A$ measurements was reported. However, this analysis differs from the others in utilizing the structure functions extracted by the experiments instead of the absolute cross-sections. Also, the uncertainties from the baseline PDFs were added on top of the experimental errors. Here, we review the results of the latest effort [9] — inspired by the findings of [5] — that sidesteps the possible experimental issues in the absolute normalization.

\section{Experimental Input And The Theoretical Framework}

The experimental neutrino cross-sections that enter to the analysis come from three independent (Fermilab and CERN) experiments: NuTeV [1], CDHSW [6] and CHORUS [7]. After applying typical cuts for the virtuality $Q^{2}>4 \mathrm{GeV}^{2}$ and for the invariant mass of the final state $W^{2}>12.25 \mathrm{GeV}^{2}, 2136 \mathrm{NuTeV}, 824 \mathrm{CHORUS}$ and $937 \mathrm{CDHSW}$ data points remain. The neutrino beam energy ranges from $E \sim 20 \mathrm{GeV}$ up to $E \sim 300 \mathrm{GeV}$. As in $[2,3,4,5]$, the theoretical calculations are performed at next-to-leading order pQCD supplemented with the SACOT prescription for the treatment of heavy quarks (in [8] a different scheme was adopted). Accordingly, we utilize the CTEQ6.6 [10] free proton PDFs, and the EPS09 nuclear modifications [11]. Corrections for electroweak radiation and target-mass effects are applied [5]. 


\section{The Normalization Procedure}

Instead of comparing the calculations directly with the absolute experimental cross-sections $\sigma_{\exp }^{v}(x, y, E)$, we form a ratio

$$
R^{v}(x, y, E) \equiv \frac{\sigma_{\exp }^{v}(x, y, E)}{\sigma_{\text {CTEQ6.6 }}^{v}(x, y, E)},
$$

where $\sigma_{\mathrm{CTEQ6} .6}^{v}(x, y, E)$ is calculated without nuclear effects in PDFs. This facilitates the interpretation of the data vs. theory comparison. As found in [5], these ratios are practically independent of $Q^{2}$ and the beam energy $E$. Therefore, we construct a following weighted average

$$
R_{\text {Average }}^{v}(x) \equiv\left(\sum_{i \in \text { fixed } x}^{N} \frac{R_{i}^{v}}{\delta_{i}}\right)\left(\sum_{i \in \text { fixed } x}^{N} \frac{1}{\delta_{i}}\right)^{-1} \pm N \times\left(\sum_{i \in \text { fixed } x}^{N} \frac{1}{\delta_{i}}\right)^{-1},
$$

where $\delta_{i}$ stands for the experimental error (divided by $\sigma_{\mathrm{CTEQ6.6}}^{V}$ ) and $N$ is the number of data points. This procedure effectively distills the average value of $R^{v}(x, y, E)$ for a given $x$-bin and gives an idea of its uncertainty. The left-hand panel of Figure 1 presents the results obtained in this way. Nonnegligible differences in the absolute normalization are visible. Especially, the NuTeV neutrino data is systematically below the rest. Apart from these normalization differences the $x$ dependence
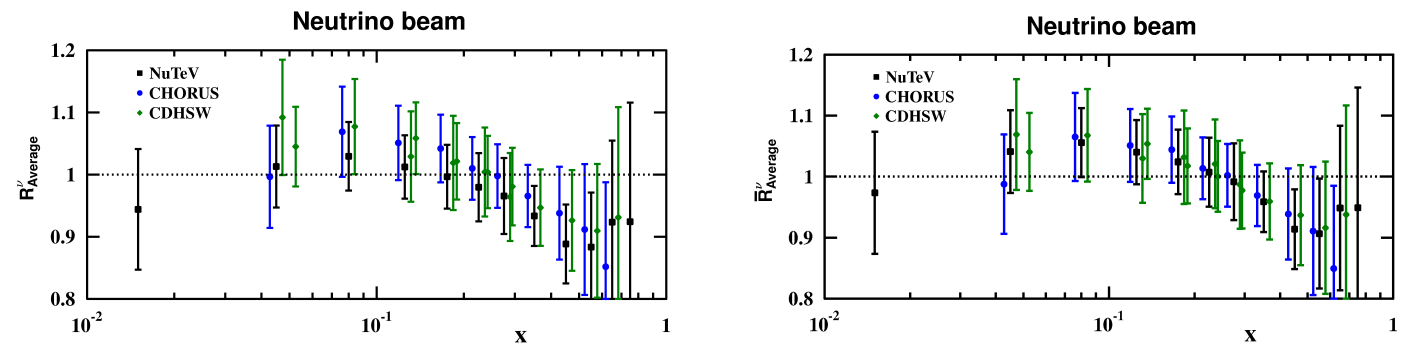

Figure 1: The neutrino data presented as $R_{\text {Average }}^{v}$ (left panel), and as $\bar{R}_{\text {Average }}^{v}$ (right panel). The CHORUS (blue circles) and CDHSW (green diamonds) data has been horizontally shifted from the NuTeV (black squares) data points.

of the $R_{\text {Average }}^{v}$ appears quite similar for each experiment. Motivated by this observation, we define

$$
I_{\exp }^{v}(E) \equiv \sum_{i \in \text { fixed } E} \sigma_{\exp , i}(x, y, E) \times B_{i}(x, y),
$$

where $B_{i}(x, y)$ is the size of the experimental $(x, y)$-bin. That is, $I_{\exp }^{v}(E)$ is an estimate for the integrated cross-section in an energy bin. Now, instead of Eq. (3.1) we consider

$$
\bar{R}^{v}(x, y, E) \equiv \frac{\sigma_{\exp }^{v}(x, y, E) / I_{\exp }^{v}(E)}{\sigma_{\text {CTEQ6.6 }}^{v}(x, y, E) / I_{\text {CTEQ6.6 }}^{v}(E)} .
$$

The right-hand panel of Figure 1 show how this simple normalization procedure seems to bring all data in perfect mutual agreement. In Figure 2, we show a comparison with the theoretical predictions from the nuclear PDFs defined in the usual manner as

$$
f_{i}^{A}\left(x, Q^{2}\right) \equiv R_{i}^{A, \operatorname{EPS} 09}\left(x, Q^{2}\right) f_{i}^{\mathrm{CTEQ} 6.6 \mathrm{M}}\left(x, Q^{2}\right) .
$$


The data points are the same as in Figure 1, and the blue band represents the uncertainty range derived using the CTEQ6.6 and EPS09 error sets. We note that large part of the CTEQ6.6 uncertainty cancels in the normalization procedure. Clearly, the nuclear PDFs can reproduce the normalized data. For the corresponding figures in the case of the antineutrino data, see [9].

\section{The Numerical Check}

We verify the consistency of these data within the CTEQ6.6 and EPS09 global fits by the Hessian reweighting technique [9] ${ }^{1}$. The method relies on the PDF uncertainty sets $S_{k}^{ \pm}$that quantify the $\Delta \chi^{2}$ neighborhood of the best fit $S_{0}$ found in a global analysis. These sets can be used to estimate the values of any PDF-dependent quantities $X_{k}$ close to the best fit as

$X_{k}[S] \approx X\left[S_{0}\right]+\left.\sum_{k} \frac{\partial X_{k}[S]}{\partial z_{k}}\right|_{S=S_{0}} z_{k} \approx X_{k}\left[S_{0}\right]+\mathbf{D}_{k} \cdot \mathbf{w}$

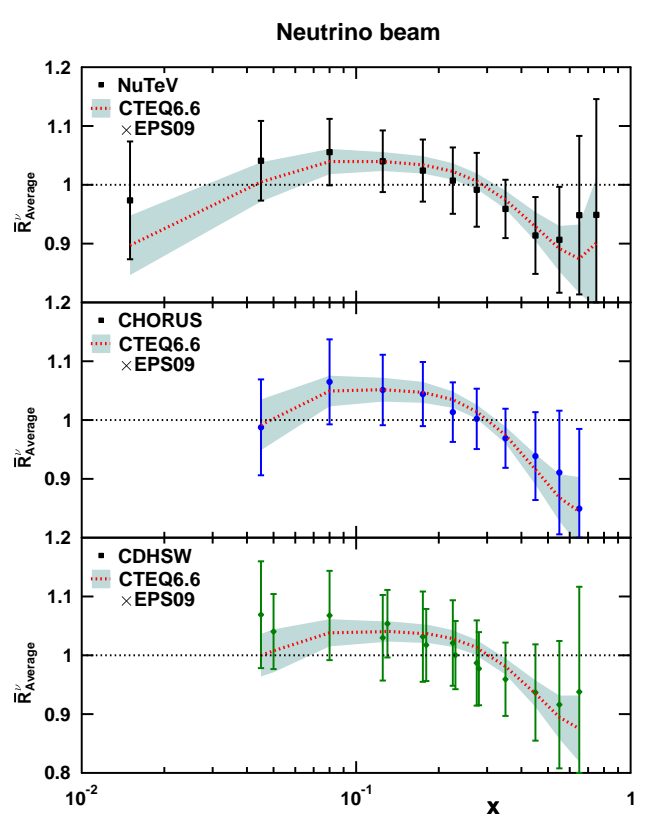

Figure 2: The experimental $\bar{R}_{\text {Average }}^{v}$ compared to the predictions from CTEQ6.6 $\otimes$ EPS09.

where $\left(D_{k}\right)_{i} \equiv\left(X_{k}\left[S_{i}^{+}\right]-X_{k}\left[S_{i}^{-}\right]\right) / 2$ and $w_{i} \equiv$ $z_{i} / \sqrt{\Delta \chi^{2}}$. Here, we take the $X_{k}$ as the neutrino cross-sections and study their compatibility within the global fits by defining a $\chi^{2}$ function by

$$
\chi^{2} \equiv \sum_{\left\{X^{\text {data }}\right\}}\left[\frac{X_{k}[S]-X_{k}^{\text {data }}}{\delta_{k}^{\text {data }}}\right]^{2}+\Delta \chi_{\text {EPS09 }}^{2} \sum_{k=1}^{15} w_{k}^{2}+\Delta \chi_{\text {CTEQ6.6 }}^{2} \sum_{k=16}^{37} w_{k}^{2},
$$

where $\Delta \chi_{\mathrm{EPS} 09}^{2}=50$ and $\Delta \chi_{\mathrm{CTEQ} 6.6}^{2}=100$. This expression is a quadratic function of the parameters $w_{i}$ and its minimum can be found by the standard methods of linear algebra. The values of the "penalty terms" $\Delta \chi_{\mathrm{EPS} 09 \backslash \mathrm{CTEQ6.6}}^{2} \sum_{k} w_{k}^{2}$ at the minimum can be used to distinguish whether the new data set is in agreement with the original fits: If $\Delta \chi_{\mathrm{EPS} 09 \backslash \mathrm{CTEQ} 6.6}^{2} \sum_{k} w_{k}^{2} \ll \Delta \chi_{\mathrm{EPS} 09 \backslash \mathrm{CTEQ6.6}}^{2}$, the new data agrees well with the original fit but if $\Delta \chi_{\mathrm{EPS} 09 \backslash \mathrm{CTEQ6.6}}^{2} \sum_{k} w_{k}^{2} \gtrsim \Delta \chi_{\mathrm{EPS} 09 \backslash \mathrm{CTEQ6} .6}^{2}$ tension clearly exists.

The Table 1 displays the key results in the case of the NuTeV data. The first column $\chi_{w=0}^{2} / N$ corresponds to the $\chi^{2}$ calculated by the central values from CTEQ6.6 $\otimes$ EPS09 (zero penalty). The normalization clearly improves the agreement. The next column $\chi_{w_{\min }}^{2} / N$ shows what happens when the minimization is performed. This naturally improves the agreement. However, this also gives rise to the penalty terms and if no normalization is applied, the penalty for the EPS09 is already close to the largest permitted value 50. With the normalization, the penalties remain small which indicates that the normalized $\mathrm{NuTeV}$ data could be added to these global fits. For the CHORUS and CDHSW data the penalties remain always very small (see [9]). In order to mimic the

\footnotetext{
${ }^{1} \mathrm{An}$ article on the relation and differences to the NNPDF reweighting [12] and MSTW work [13] will appear later.
} 


\begin{tabular}{r|cccc||cc}
\multicolumn{5}{c||}{ All CTEQ6.6 and EPS09 error sets } & \multicolumn{2}{c}{ Only EPS09 error sets } \\
NuTeV & $\chi_{w=0}^{2} / N$ & $\chi_{w_{\min }}^{2} / N$ & EPS09-penalty & CTEQ-penalty & $\chi_{w_{\min }}^{2} / N$ & EPS09-penalty \\
Normalization & 0.84 & 0.77 & 13.9 & 35.4 & 0.81 & 33.8 \\
No normalization & 1.04 & 0.90 & 40.3 & 42.5 & 0.94 & 77.4
\end{tabular}

Table 1: The $\chi^{2} / N$ for the NuTeV data and the EPS09 and CTEQ6.6 penalties. See the text for details.

analysis of Ref. [4] where an incompatibility was found, we freeze the CTEQ6.6 to its central value. The corresponding results (now the CTEQ penalty is zero) values are shown in the two right-most panels. Without the normalization, the EPS09-penalty is almost 80 - clearly above the permitted 50. That is, we would reach the same conclusion as the authors of Ref. [4].

\section{Conclusion}

As a summary, we have demonstrated that independent neutrino data sets seem to disagree in the absolute normalization. Especially, the $\mathrm{NuTeV}$ data show a difference with the rest. We propose to normalize the data by the corresponding integrated cross-section which appears to largely dispose the differences among the data sets. The Hessian reweighting technique is used to study the consistency with the present nuclear PDFs, and a good agreement is found when the normalization procedure is considered. Without the normalization we recover the contradictory results of Ref. [4].

\section{References}

[1] M. Tzanov et al. [NuTeV Collaboration], Phys. Rev. D 74 (2006) 012008 [arXiv:hep-ex/0509010].

[2] I. Schienbein, J. Y. Yu, C. Keppel, J. G. Morfin, F. Olness and J. F. Owens, Phys. Rev. D 77 (2008) 054013 [arXiv:0710.4897 [hep-ph]].

[3] I. Schienbein, J. Y. Yu, K. Kovarik, C. Keppel, J. G. Morfin, F. Olness and J. F. Owens, Phys. Rev. D 80 (2009) 094004 [arXiv:0907.2357 [hep-ph]].

[4] K. Kovarik et al., Phys. Rev. Lett. 106 (2011) 122301 [arXiv:1012.0286 [hep-ph]].

[5] H. Paukkunen and C. A. Salgado, JHEP 1007 (2010) 032 [arXiv:1004.3140 [hep-ph]].

[6] J. P. Berge et al., Z. Phys. C 49 (1991) 187.

[7] G. Onengut et al. [CHORUS Collaboration], Phys. Lett. B 632 (2006) 65.

[8] D. de Florian, R. Sassot, P. Zurita and M. Stratmann, Phys. Rev. D 85 (2012) 074028

[arXiv:1112.6324 [hep-ph]].

[9] H. Paukkunen and C. A. Salgado, Phys. Rev. Lett. 110 (2013) 212301 [arXiv:1302.2001 [hep-ph]].

[10] P. M. Nadolsky et al., Phys. Rev. D 78 (2008) 013004 [arXiv:0802.0007 [hep-ph]].

[11] K. J. Eskola, H. Paukkunen and C. A. Salgado, JHEP 0904 (2009) 065 [arXiv:0902.4154 [hep-ph]].

[12] R. D. Ball et al. [NNPDF Collaboration], Nucl. Phys. B 849 (2011) 112 [Erratum-ibid. B 854 (2012) 926] [Erratum-ibid. B 855 (2012) 927] [arXiv:1012.0836 [hep-ph]].

[13] G. Watt and R. S. Thorne, JHEP 1208 (2012) 052 [arXiv:1205.4024 [hep-ph]]. 